\title{
Mitigating effects of external ventricular drain usage in the management of severe head injury
}

\author{
Andrew Maas \\ Received: 19 April 2013 / Accepted: 22 April 2013 /Published online: 15 May 2013 \\ (C) Springer-Verlag Wien 2013
}

In this issue of Acta Neurochirurgica, Bhargava et al. [1] report on the efficacy of external ventricular drainage of cerebrospinal fluid (CSF) for the management of raised intracranial pressure (ICP) in patients with traumatic brain injury (TBI) refractory to conventional medical management. In contrast to clinical practice in many centres, in this study CSF drainage via external ventricular drain (EVD) was used as a third-tier therapy rather than as one of the first approaches to the management of raised ICP. Only 2 out of 16 patients treated in this manner required additional third-tier therapies (decompressive craniectomy or barbiturates therapy). In patients with raised ICP refractory to medical management primarily treated by either decompression or barbiturates therapy, two patients also required additional placement of an EVD. Thus, external CSF drainage, when used as third-tier therapy, would appear to be equally effective in controlling raised ICP as barbiturates or a decompressive craniectomy. This would seem to make a strong case for advocating use of a ventricular catheter for ICP monitoring and when required external drainage of CSF.

Previous reports have likewise provided evidence in support of CSF drainage for controlling ICP. In a relatively small cohort study on $20 \mathrm{TBI}$ patients, Lescot et al. [2] showed that continuous CSF drainage was effective not only in lowering ICP levels but also in decreasing treatment intensity. Shore et al. [3] reported that continuous drainage was more efficient than intermittent CSF drainage in paediatric TBI.

The finding that external ventricular drainage of CSF constitutes a useful ICP-lowering manoeuvre in patients with raised ICP refractory to other measures is not new. Timofeev et al. [4] reported similar findings in 2008 and further demonstrated significant improvements in cerebral

\footnotetext{
A. Maas $(\bowtie)$

Department of Neurosurgery, Antwerp University Hospital, Wilrijkstraat 10, 2650 Edegem, Belgium

e-mail: andrew.maas@uza.be
}

perfusion pressure, brain tissue oxygen and energy metabolism as evidenced by lactate/pyruvate ratios observed in microdialysis studies.

The study by Bhargava et al. [1] now lends further support for CSF drainage in TBI. Nevertheless, the study has its limitations and interpretation should be made with caution. Besides its retrospective nature, and the lack of randomisation, there are two aspects related to this study which are of particular concern. First, the authors focus on 33 patients with TBI and raised ICP, refractory to conventional medical therapy. All these patients had been diagnosed with severe TBI but only one death is reported in this cohort, which would appear to be unbelievably low for a population of severe TBI with raised ICP. The only possible explanations are either that the institution is incredibly good at treating TBI or that in some way the patient population is selected. The latter would appear more likely. Secondly, and most importantly, the authors state that patients with sustained ICP increases were considered for the RESCUEicp trial (www.rescueicp.com). As can be read clearly from Fig. 1, patients treated by additional CSF drainage were excluded from participation in the RESCUEicp study. Consequently the local procedures imply a selection in patients considered for randomisation into RESCUEicp. Introducing a selection bias in this way jeopardises the principle of equipoise underpinning RESCUEicp. Furthermore, this selection illustrates how approaches to ICP monitoring and its treatment may confound consistent use of enrolment criteria, not only across study centres but even within a single centre.

Although the study reported by Bhargava et al. [1] is interesting and provides evidence in support of CSF drainage, these two concerns are such that they leave me with an uncomfortable feeling, as in my opinion they are illustrative of possible risks and consequences of selection bias. It is highly likely that selection bias occurred within the cohort study and local study 
procedures further introduced a selection bias into an ongoing multicentre randomised controlled trial.

\section{Conflicts of interest None.}

\section{References}

1. Bhargava D, Alade A, Ellamushi H, Yeh J, Hunter Rl (2013) Mitigating effects of external ventricular drain usage in the management of severe head injury. Acta Neurochir (Wein). doi:10.1007/ s00701-013-1735-8
2. Lescot T, Boroli F, Reina V, Chauvet D, Boch AL, Puybasset L (2012) Effect of continuous cerebrospinal fluid drainage on therapeutic intensity in severe traumatic brain injury. Neurochirurgie $58: 235-240$

3. Shore PM, Thomas NJ, Clark RS, Adelson PD, Wisniewski SR, Janesko KL, Bayir H, Jackson EK, Kochanek PM (2004) Continuous versus intermittent cerebrospinal fluid drainage after severe traumatic brain injury in children: effect on biochemical markers. J Neurotrauma 21:1113-1122

4. Timofeev I, Dahyot-Fizelier C, Keong N, Nortje J, Al-Rawi PG, Czosnyka M, Menon DK, Kirkpatrick PJ, Gupta AK, Hutchinson PJ (2008) Ventriculostomy for control of raised ICP in acute traumatic brain injury. Acta Neurochir Suppl 102:99-104 